\title{
Combining biomedical and agricultural research: is an IACUC necessary for pilot data collection?
}

\author{
Jerald Silverman, DVM
}

Riya Ramos, an animal husbandry professor at Great Eastern University, bred hybrids of domestic cattle and American bison. Her goal was to develop rapid growing, wellmarbled cattle that also would produce low fat milk, rather than the typically sweet-tasting high fat milk of the hybrids. She began a search to identify a genetic sequence that might be indicative of a low-fat producing cow. As her studies progressed she discovered that in the very few low milk fat producing cows she was able to identify, there was a linked genetic sequence previously reported as being a variant of the prion protein gene. Nevertheless, not one of her animals ever exhibited bovine spongiform encephalopathy, a prion-associated disease. Intrigued, yet preferring to remain focused on her area of expertise, Ramos brought her finding to Jack Mason, a neurologist in the university's veterinary college, and they tentatively agreed to develop a collaborative pilot study looking at additional genetic sequences from high and low milk fat producing animals.

When Mason told Ramos that an IACUC protocol would have to be submitted for approval before any work on prion disease could progress, Ramos balked. Although she had heard of IACUCs, she never had interacted with one and did not want a committee approving what she could or could not do with her animals. In Ramos's mind she was doing agricultural research, not biomedical research. She said that she would be glad to give Mason any extra blood, milk, or tissue samples from those she collected for her own research, but that was as much as she was willing to do. Mason thought that if the IACUC would approve a protocol that listed only him as a biomedical research participant, he could certainly get sufficient preliminary data from those samples. When he broached the issue with the IACUC chair, the chair said he didn't think IACUC approval was even necessary because he would be using extra samples that Ramos was not going to use for her own research.

What is your opinion? Is IACUC approval needed for the pilot study to progress? If it is, what conditions might the IACUC establish so that Ramos could do her agricultural work and Mason could use the same animals for his biomedical research?

\section{RESPONSE}

\section{No IACUC approval necessary, but inspect potential risks}

\section{Lori R Hill}

Ramos has been conducting her work without IACUC approval. This fact leads us to several conclusions: (1) The study is not funded by the NIH (subject to PHS Policy) because Ramos did not have to obtain IACUC approval in order to receive funding. ${ }^{1}$ One can also assume that the institution's OLAW Assurance is not applicable to research activities that are not funded by the NIH. (2) According to the AWAR: "Animal....This term excludes....livestock or poultry used or intended for use for improving animal nutrition, breeding, management, or production efficiency, or for improving the quality of food or fiber." ${ }^{2}$ Ramos' studies are therefore excluded from USDA oversight (and thus the requirement for IACUC review). (3) If the University is accredited by AAALAC, the agricultural portion of the animal care and use program is not included, because the Guides for the Care and Use of Laboratory Animals and for the Care and Use of Agricultural Animals in Research and Teaching would have required an IACUC protocol. ${ }^{3,4}$ (4) Ramos' funding agency does not independently require IACUC review.

The AWAR states that "research facility means any school..., institution, organization, or person that uses or intends to use live animals in research, tests, or experiments." 2 The PHS Policy defines an animal as "any live, vertebrate animal...." 1 AAALAC International defines a laboratory animal as "any live vertebrate animal... used or intended for use in research, testing, or teaching." ${ }^{5}$ Mason's proposed studies involve the use of biological materials that would normally be discarded (not live animals). Therefore, his project is not subject to the requirements of these entities, and IACUC review is not mandatory.

There are several circumstances that could require Mason to obtain IACUC approval. His funding agency could require IACUC review, or the institution could choose to require review of the use of biological materials. An institutional requirement seems unlikely since the IACUC Chair doesn't think a protocol is required. However, for consistency and for public relations purposes, review of the activity might be prudent.

Another aspect of this scenario that raises concern is the handling of excreta and tissue that contain a linked genetic sequence previously reported as being a variant of the prion protein gene. Although none of Ramos' animals ever exhibited bovine spongiform encephalopathy (BSE), there could still be a zoonotic risk to personnel. Bison are susceptible to BSE. ${ }^{6}$ This disease has a long incubation period ( $4-5$ years), so cattle or bison could be infected without exhibiting clinical signs. According to the BMBL: 